Pacific

Journal of

Mathematics

SPACELIKE S-WILLMORE SPHERES

IN LORENTZIAN SPACE FORMS

PENG WANG 


\title{
SPACELIKE S-WILLMORE SPHERES IN LORENTZIAN SPACE FORMS
}

\author{
PENG WANG
}

\begin{abstract}
We show that spacelike $S$-Willmore surfaces are the only spacelike Willmore surfaces with a duality in Lorentzian space forms. We obtain a classification of S-Willmore spheres in Lorentzian conformal space forms. Such a sphere must be congruent to either a complete spacelike stationary $(\vec{H}=0)$ surface in $R_{1}^{n}$; a super-Willmore sphere in $S^{2 m+2}$; or a polar transform of a $(j-1)$ isotropic complete spacelike stationary $(\vec{H}=0)$ surface in $R_{1}^{2 j+2}$. We also show that all Willmore spheres in $Q_{1}^{4}$ are conformal to a complete spacelike stationary surface in $R_{1}^{4}$.
\end{abstract}

\section{Introduction}

The best-known results in global study of submanifolds in space forms are about minimal and CMC spheres, for example, the classical Hopf theorem and Chern's work [1970]. Later this study was extended to Willmore 2-spheres in Möbius geometry [Bryant 1984; Ejiri 1988b; Musso 1990; Montiel 2000] and Lorentzian conformal geometry [Alías and Palmer 1996; Ma and Wang 2008]. In this paper we generalize some of these results for spacelike S-Willmore spheres in Lorentzian space forms.

Blaschke [1929] and Thomsen [1923] began the study of Willmore surfaces, and derived the dual property. Bryant [1984] rediscovered the duality theorem and used it in classifying all Willmore spheres in $S^{3}$. Then Ejiri [1988b] found that the duality theorem does not hold for general Willmore surfaces in $S^{n}$ and showed that S-Willmore surfaces are the only Willmore surfaces with a dual Willmore surfaces in $S^{n}$. He also classified all S-Willmore spheres in $S^{n}$. In the case $n=4$, Willmore spheres are automatically S-Willmore, a fact also studied in [Montiel 2000] and [Musso 1990]. When the case $n>4$, Ma [2006] showed that there exists another category of transforms, the adjoint transforms, that generalize dual surfaces. By

MSC2000: primary 53A30; secondary 53B30.

Keywords: spacelike S-Willmore surfaces, spacelike S-Willmore sphere, stationary surfaces, duality theorem.

Supported by Program for Young Excellent Talents in Tongji University and the Tianyuan Foundation of China, grant 10926112. 
using adjoint transforms, he obtained partial results concerning the classification of Willmore 2-sphere in $S^{n}$ [Ma 2005].

In the Lorentzian case, Alías and Palmer [1996] showed that the duality theorem holds for spacelike Willmore surfaces in 3-dimensional Lorentzian space forms, and they proved that the only spacelike Willmore 2-sphere is the round sphere. In higher-dimensional Lorentzian space forms, the duality theorem does not hold in general either [Ma and Wang 2008]. While we can define the so-called polar transforms for surfaces in 4-dimensional Lorentzian space forms, such transforms keep the Willmore property. We also obtained the classification theorem:

Theorem A [Ma and Wang 2008]. Any spacelike Willmore 2-sphere in $Q_{1}^{4}$ is either congruent to a complete spacelike stationary surface (that is, $H=0$ ) in $R_{1}^{4}$, or a polar surface of such a surface.

Theorem B [Ejiri 1988b]. Every S-Willmore 2-sphere y $: S^{2} \rightarrow S^{n}$ must be conformally equivalent to a super $S$-Willmore surface or a complete minimal surface in $R^{n}$ with its dual surface reducing to a point.

For the Lorentzian case, we obtain the duality theorem too. Then we generalize the notion of polar surfaces and derive the classifications of S-Willmore spheres:

Theorem C. Suppose y: $S^{2} \rightarrow Q_{1}^{n}$ is a spacelike $S$-Willmore 2-sphere. Then up to conformal equivalence, it is described in one of the following:

(i) $y$ is congruent to a complete spacelike stationary surface (that is, $\vec{H}=0$ ) in $R_{1}^{n}$, and its dual surface reduces to a point.

(ii) $y$ is a super $S$-Willmore surface in some Riemannian sphere $S^{2 m} \subset Q_{1}^{n}$.

(iii) $y$, together with its dual surface, is a polar surface of an $(m-1)$-isotropic complete spacelike stationary surface in $R_{1}^{2 m}$, and all are in some subspace $Q_{1}^{2 m} \subset Q_{1}^{n}$.

Here the space $Q_{1}^{n}$ is the conformal compactification of Lorentzian space forms. Theorem $\mathrm{C}$ is proved by constructing holomorphic forms on $S^{2}$, which must vanish. Then it reduces to three cases. The first two cases are the same as in $S^{n}$. The third is that it is a full totally isotropic sphere in $Q_{1}^{2 m} \subset Q_{1}^{n}$. There are two difficulties in the proof. The first is to show that the holomorphic forms are globally defined in $S^{2}$. The second is to describe the totally isotropic sphere in $Q_{1}^{2 m}$. Another problem is the existence of surfaces in the case (iii). When $n=4$, we prove that it must be a round sphere. When $n>4$, in $R_{1}^{6}$ we give some examples of surfaces belonging to case (i) and (iii). As to surfaces belonging to case (iii), we still know little about them.

This paper is organized as follows. In Section 2, we review the notion of $Q_{1}^{n}$, the general theory of spacelike surfaces, the description of Willmore and S-Willmore 
surfaces, and the duality theorem for S-Willmore surfaces. In Section 3, we construct holomorphic forms on Willmore 2-spheres and reduce the problem to describing the totally isotropic sphere in $Q_{1}^{2 m}$. In Section 4, we derive the notion of polar surfaces, showing that a totally isotropic sphere in $Q_{1}^{2 m}$ is either congruent to a totally isotropic minimal surface in $\mathbb{R}_{1}^{2 m}$ or to a polar surface of $(m-1)$-isotropic minimal surfaces in $\mathbb{R}_{1}^{2 m}$. We also exhibit an S-Willmore sphere conformal to a totally isotropic minimal surface in $R_{1}^{6}$. In the last section, we show that the spheres in the second case of Theorem A must be round spheres.

In the sequel $y: M \rightarrow Q_{1}^{n}$ will always denote a smooth spacelike immersion from an oriented surface $M$ unless it is explicitly stated otherwise.

\section{Spacelike surfaces in Lorentzian conformal geometry}

Here we will review the conformal geometry of $Q_{1}^{n}$ and the spacelike surface theory in $Q_{1}^{n}$. For further details, see [Alías and Palmer 1996; Ma and Wang 2008]. Our treatment here follows the surface theory in [Burstall et al. 2002].

Let $\mathbb{R}_{2}^{n+2}$ be the space $\mathbb{R}^{n+2}$ equipped with the quadric form

$$
\langle x, x\rangle=\sum_{1}^{n} x_{i}^{2}-x_{n+1}^{2}-x_{n+2}^{2},
$$

with $C_{1}^{n+1}$ its light cone. The projective light cone

$$
Q_{1}^{n}=\left\{[x] \in \mathbb{R} P^{n+1} \mid x \in C_{1}^{n+1} \backslash\{0\}\right\}
$$

is equipped with a Lorentzian metric induced from the projection $S^{n-1} \times S^{1} \subset$ $C_{1}^{n+1} \backslash\{0\} \rightarrow Q_{1}^{n}$ together with a Lorentzian metric $g\left(S^{n-1}\right) \oplus\left(-g\left(S^{1}\right)\right)$, where $g\left(S^{n-1}\right)$ and $g\left(S^{1}\right)$ are standard metrics on $S^{n-1}$ and $S^{1}$. So there is a conformal Lorentzian metric $[h]$ on $Q_{1}^{n}$. The conformal group of $\left(Q_{1}^{n},[h]\right)$ is exactly the orthogonal group $O(n, 2) /\{ \pm 1\}$ of $\mathbb{R}_{2}^{n+2}$, acting on $Q_{1}^{n}$ by

$$
T([x])=[x T] \text { for } T \in O(n, 2) .
$$

The three $n$-dimensional Lorentzian space forms with constant sectional curvature $c=0,+1,-1$ can be conformally embedded as a proper subset of $Q_{1}^{n}$. For more details about Lorentzian space forms, see [O'Neill 1983].

Now we focus on a spacelike surface $y: M \rightarrow Q_{1}^{n}$, with $M$ a Riemann surface. Let $U \subset M$ be an open subset. A local lift of $y$ is a map $Y: U \rightarrow C_{1}^{n+1} \backslash\{0\}$ such that $\pi \circ Y=y$. Two different local lifts differ by a scaling, so their induced metrics are conformal. We call $y$ a conformal spacelike surface if the induced metric is conformal, that is, $\left\langle Y_{z}, Y_{z}\right\rangle=0$ and $\left\langle Y_{z}, Y_{\bar{z}}\right\rangle>0$ for any local lift $Y$ and any complex coordinate $z$ on $M$. Then there is a decomposition $M \times \mathbb{R}_{2}^{n+2}=V \oplus V^{\perp}$, where $V=\operatorname{span}\left\{Y, \mathrm{~d} Y, Y_{z \bar{z}}\right\}$ is a Lorentzian rank-4 subbundle independent of the choice 
of $Y$ and $z \cdot V^{\perp}$ is also a Lorentzian subbundle, called the conformal normal bundle of $y$ in $Q_{1}^{n}$. Their complexifications are denoted respectively by $V_{\mathbb{C}}$ and $V_{\mathbb{C}}^{\perp}$.

Fix a local coordinate $z$. There is a local lift $Y$ satisfying $|\mathrm{d} Y|^{2}=|\mathrm{d} z|^{2}$, called the canonical lift (with respect to $z$ ). Choose a frame $\left\{Y, Y_{z}, Y_{\bar{z}}, N\right\}$ of $V_{\mathbb{C}}$, where $N \in \Gamma(V)$ is uniquely determined by

$$
\left\langle N, Y_{z}\right\rangle=\left\langle N, Y_{\bar{z}}\right\rangle=\langle N, N\rangle=0 \quad \text { and } \quad\langle N, Y\rangle=-1 .
$$

Definition 2.1 [Bryant 1984; Ejiri 1988b; Alías and Palmer 1996; Ma and Wang 2008]. For a conformally immersed surface $y: M \rightarrow Q_{1}^{n}$ with canonical lift $Y$ (with respect to a local coordinate $z$ ), we define

$$
G:=Y \wedge Y_{u} \wedge Y_{v} \wedge N=-2 i \cdot Y \wedge Y_{z} \wedge Y_{\bar{z}} \wedge N, \quad \text { where } z=u+i v .
$$

Here $N \equiv 2 Y_{z \bar{z}}(\bmod Y)$ is the frame vector determined in $(1)$. Note that $\langle G, G\rangle=1$ and that $G$ is well defined. We call $G: M \rightarrow G_{3,1}\left(\mathbb{R}_{2}^{n+2}\right)$ the conformal Gauss map of $y$.

Given frames as above, and noting that $Y_{z z}$ is orthogonal to $Y, Y_{z}$ and $Y_{\bar{z}}$, there must be a complex function $s$ and a section $\kappa \in \Gamma\left(V_{\mathbb{C}}^{\perp}\right)$ such that

$$
Y_{z z}=-\frac{1}{2} s Y+\kappa .
$$

This defines two basic invariants $\kappa$ and $s$ depending on coordinates $z$, the conformal Hopf differential and the Schwarzian of $y$; for discussion, see [Burstall et al. 2002; Ma 2005]. Let $D$ denote the normal connection and $\psi \in \Gamma\left(V_{\mathbb{C}}^{\perp}\right)$ any section of the normal bundle. Then the structure equations can be given as

$$
\begin{aligned}
Y_{z z} & =-\frac{1}{2} s Y+\kappa, \\
Y_{z \bar{z}} & =-\langle\kappa, \bar{\kappa}\rangle Y+\frac{1}{2} N, \\
N_{z} & =-2\langle\kappa, \bar{\kappa}\rangle Y_{z}-s Y_{\bar{z}}+2 D_{\bar{z}} \kappa, \\
\psi_{z} & =D_{z} \psi+2\left\langle\psi, D_{\bar{z}} \kappa\right\rangle Y-2\langle\psi, \kappa\rangle Y_{\bar{z}} .
\end{aligned}
$$

The conformal Gauss, Codazzi and Ricci equations as integrable conditions are

$$
\begin{aligned}
s_{\bar{z}} & =6\left\langle\kappa, D_{z} \bar{\kappa}\right\rangle+2\left\langle D_{z} \kappa, \bar{\kappa}\right\rangle, \\
0 & =\operatorname{Im}\left(D_{\bar{z}} D_{\bar{z}} \kappa+\frac{1}{2} \bar{s} \kappa\right), \\
R_{\bar{z} z}^{D} & =D_{\bar{z}} D_{z} \psi-D_{z} D_{\bar{z}} \psi=2\langle\psi, \kappa\rangle \bar{\kappa}-2\langle\psi, \bar{\kappa}\rangle \kappa .
\end{aligned}
$$

These are parallel to the theory in [Burstall et al. 2002]. The conformal Hopf differentials play an important role in the research of Willmore surfaces. To see this, we first give the transformation formula of $\kappa$. For another complex coordinate $w$, $Y_{1}=Y \cdot|d w / d z|$ is a canonical lift with respect to $w$. So the corresponding Hopf 
differential $\kappa_{1}$ with respect to $\left(Y_{1}, w\right)$ is

$$
\kappa_{1}=\kappa \cdot\left(\frac{d z}{d w}\right)^{2} \cdot\left|\frac{d z}{d w}\right|^{-1} .
$$

Direct computation using (2) and (4) shows that $G$ induces a conformal-invariant metric

$$
g:=\frac{1}{4}\langle\mathrm{~d} G, \mathrm{~d} G\rangle=\langle\kappa, \bar{\kappa}\rangle|d z|^{2}
$$

on $\mathrm{M}$. This metric might be positive definite, negative definite, or degenerate, depending on the sign of $\langle\kappa, \bar{\kappa}\rangle$. We define the Willmore functional and Willmore surfaces by using this metric.

Definition 2.2. The Willmore functional of $y$ is defined as the area of $M$ with respect to the metric above:

$$
W(y):=2 i \int_{M}\langle\kappa, \bar{\kappa}\rangle d z \wedge d \bar{z} .
$$

An immersed surface $y: M \rightarrow Q_{1}^{n}$ is called a Willmore surface if it is a critical surface of the Willmore functional with respect to any variation of the map $y: M \rightarrow Q_{1}^{n}$.

By (4), it is easy to see that $W(y)$ is well defined. The following characterization of Willmore surfaces is similar to the one in the codimension-1 case [Alías and Palmer 1996] as well to the one in Möbius geometry [Bryant 1984; Burstall et al. 2002; Ejiri 1988b; Wang 1998].

Theorem 2.3. For a conformal spacelike surface $y: M \rightarrow Q_{1}^{n}$, the following four conditions are equivalent:

(i) y is Willmore.

(ii) The conformal Gauss map $G$ is a harmonic map into $G_{3,1}\left(\mathbb{R}_{2}^{n+2}\right)$.

(iii) The conformal Hopf differential $\kappa$ of $y$ satisfies the Willmore condition

$$
D_{\bar{z}} D_{\bar{z}} \kappa+\frac{1}{2} \bar{s} \kappa=0,
$$

which is a stronger condition than the conformal Codazzi equation (3).

The proof of this theorem is completely the same as in Möbius geometry (we refer readers to [Ma 2005; Wang 1998]).

Now we introduce the so-called S-Willmore surfaces, the Willmore surfaces with dual surfaces (see the next section). See [Ejiri 1988b] and [Ma 2005] for their counterparts in Möbius geometry. 
Definition 2.4. A conformal Willmore surface $y: M \rightarrow Q_{1}^{n}$ is called a $S$-Willmore surface if it satisfies $D_{\bar{z}} \kappa \| \kappa$, that is,

$$
D_{\bar{z}} \kappa=-\frac{1}{2} \bar{\mu} \kappa
$$

for some local function $\mu$ when $\kappa \neq 0$ (that is, when $\kappa$ is nonumbilical). Here the conformal Hopf differential $\kappa$ is defined in (2).

Note that a conformal surface satisfying $D_{\bar{z}} \kappa \| \kappa$ is not necessary Willmore; see [Ma and Wang 2008].

Theorem 2.5. Any spacelike $S$-Willmore surface $[Y]: M \rightarrow Q_{1}^{n}$ has a dual $S$ Willmore surface $[\hat{Y}]$, meaning they share the same central sphere and the same complex coordinates (and hence the same normal bundle and normal connection). The dual surface $[\hat{Y}]$ degenerates to a point if and only if $[Y]$ is congruent to a spacelike stationary $(H=0)$ surface in $R_{1}^{4}$. Conversely, a spacelike Willmore surface in $Q_{1}^{n}$ with a dual Willmore surface must be an $S$-Willmore surface.

Proof. If $[Y]$ is totally umbilical, the proof is trivial. If $[Y]$ is not totally umbilical, by using that $[Y]$ is real analytic (see [Ejiri 1988b; Alías and Palmer 1996]), we see that $M_{0}=M \backslash\{$ umbilical points $\}$ is an open dense subset of $M$.

For an S-Willmore surface $y: M \rightarrow Q_{1}^{n}$ with canonical lift $Y$ (with respect to a local coordinate $z$ ), suppose that $D_{\bar{z}} \kappa=-\frac{1}{2} \bar{\mu} \kappa$ for some local function $\mu$. Set

$$
\hat{Y}=N+\bar{\mu} Y_{z}+\mu Y_{\bar{z}}+\frac{1}{2}|\mu|^{2} Y .
$$

We will show it is just the dual S-Willmore surface of $y$.

In fact, substituting $D_{\bar{z}} \kappa=-\frac{1}{2} \bar{\mu} \kappa$ into the Willmore equation (5) shows that

$$
\mu_{z}-\frac{1}{2} \mu^{2}-s=0
$$

which leads to

$$
\hat{Y}_{z}=\frac{1}{2} \mu \hat{Y}+\rho\left(Y_{z}+\frac{1}{2} \mu Y\right), \quad \text { where } \rho=\bar{\mu}_{z}-2\langle\kappa, \bar{\kappa}\rangle .
$$

So

$$
\begin{aligned}
& \hat{Y}_{z \bar{z}}=\left(\frac{1}{2} \mu \hat{Y}+\rho\left(Y_{z}+\frac{1}{2} \mu Y\right)\right)_{\bar{z}}=(\cdots) \hat{Y}+(\cdots) \hat{Y}_{z}+(\cdots) \hat{Y}_{\bar{z}}+(\cdots) Y, \\
& \operatorname{span}_{\mathbb{C}}\left\{Y, Y_{z}, Y_{\bar{z}}, Y_{z \bar{z}}\right\}=\operatorname{span}_{\mathbb{C}}\left\{\hat{Y}, \hat{Y}_{z}, \hat{Y}_{\bar{z}}, \hat{Y}_{z \bar{z}}\right\} .
\end{aligned}
$$

Since $y$ and $[\hat{Y}]$ have common central sphere, complex coordinates, and harmonic conformal Gauss map, $[\hat{Y}]$ is a Willmore surface by Theorem 2.3.

From the definition of $\rho$, using (3) and (8), we see that

$$
\rho_{\bar{z}}=\bar{\mu}_{z \bar{z}}-2\left\langle D_{\bar{z}} \kappa, \bar{\kappa}\right\rangle-2\left\langle\kappa, D_{\bar{z}} \bar{\kappa}\right\rangle=\bar{\mu} \rho .
$$


So either $\rho$ must have isolated zero points or $\rho \equiv 0$; see [Chern 1970, Section 4]. If $\rho \equiv 0$, then $\hat{Y}$ reduces to a point. If $\rho \neq \equiv$, then $\tilde{Y}=\hat{Y} /|\rho|$ is a canonical lift with respect to $z$. Direct computation leads to

$$
\tilde{\kappa}=\tilde{Y}_{z z} \bmod \tilde{Y}=(\rho /|\rho|) \kappa .
$$

With the same normal bundle as $y$ it is easy to see that $\tilde{D}_{z}=D_{z}$, where $\tilde{D}_{z}$ is the normal connection of $\tilde{Y}$. So $\tilde{D}_{\bar{z}} \tilde{\kappa} \| \tilde{\kappa}$, showing that $[\hat{Y}]$ is S-Willmore.

Then Proposition 2.6 finishes the proof on $M_{0}$.

Now consider the remaining points. The function $\mu$ is analytic satisfying (8) except at the umbilical points. So for any $p \in M \backslash\left\{M_{0}\right\}$, either $\mu$ extends to a finite number on $p$ or goes to infinity on $p$. For the first case, we still define $\hat{Y}$ by (7); for the second case, we define $\hat{Y}$ by

$$
\left[\hat{Y}_{p}\right]=\lim _{\mu \rightarrow \infty}\left[\frac{1}{|\mu|^{2}}\left(N+\bar{\mu} Y_{z}+\mu Y_{\bar{z}}+\frac{1}{2}|\mu|^{2} Y\right)\right]=\left[Y_{p}\right]
$$

So we extend the definition of $\hat{Y}$ continuously to the umbilical points, finishing the proof of our theorem.

Proposition 2.6. If $\hat{Y}$ reduces to a point, then $y=[Y]$ is congruent to a complete spacelike stationary surface (that is, $\vec{H}=0$ ) in $R_{1}^{n}$, and its dual surface reduces to a point.

Proof. If $\hat{Y}$ reduces to a point, by applying a transformation $T \in O(n, 2)$ if necessary, we can set

$$
\hat{Y}=(1,0, \ldots, 0,1) \quad \text { and } \quad Y=\left(\frac{1}{2}(-1+\langle u, u\rangle), u, \frac{1}{2}(1+\langle u, u\rangle)\right),
$$

where $u: U \rightarrow R_{1}^{n}$. Let $z$ be an arbitrary complex coordinate. Then we have

$$
Y_{z \bar{z}}=a Y+\left\langle Y_{z}, Y_{\bar{z}}\right\rangle N \quad \text { and } \quad \hat{Y}=N+\bar{\mu} Y_{z}+\mu Y_{\bar{z}}+\left\langle Y_{z}, Y_{\bar{z}}\right\rangle|\mu|^{2} Y,
$$

where $a, \mu$ are two functions. It is easy to see that $\hat{Y}_{z}=-\mu\left\langle Y_{z}, Y_{\bar{z}}\right\rangle \hat{Y}+\cdots$. So $\mu \equiv 0$ and $Y_{z \bar{z}}=a Y+\left\langle Y_{z}, Y_{\bar{z}}\right\rangle \hat{Y}$. Replacing by $u$ leads to

$$
\left(\left\langle u_{z \bar{z}}, u\right\rangle, u_{z \bar{z}},\left\langle u_{z \bar{z}}, u\right\rangle\right)=\left(\frac{1}{2}(-a+a\langle u, u\rangle), a u, \frac{1}{2}(a+a\langle u, u\rangle)\right) .
$$

This implies $a \equiv 0$ and $u_{z \bar{z}} \equiv 0$. So $u$ is a stationary surface in $\mathbb{R}_{1}^{n}$, and $y=[Y]$ belongs to class (i) in Theorem $\mathrm{C}$.

\section{S-Willmore spheres in $Q_{1}^{n}$}

Let $y: S^{2} \rightarrow Q_{1}^{n}$ be an S-Willmore sphere with $(Y, z)$ as above and with its dual surface $\hat{Y}$ as in (7). First, we will introduce the notion of $m$-isotropic surfaces and give some properties of such surfaces. Then we define holomorphic forms for $m$-isotropic S-Willmore spheres by a lemma. With these vanishing forms, we 
prove the first two cases of Theorem $\mathrm{C}$ and leave the discussion of the last case to Section 4.

Definition 3.1. An immersed spacelike surface $y: M \rightarrow Q_{1}^{n}$ is called an $m$-isotropic surface if it satisfies

$$
\left\langle Y_{z}^{(i)}, Y_{z}^{(j)}\right\rangle=0 \text { for } i+j \leq 2 m+1 .
$$

Here $Y_{z}^{(i)}$ denotes the $i$-th derivative of $Y$ with respect to $z$. If the equations above hold for any $i$ and $j$, we call $y$ totally isotropic.

It is direct to verify this notion is well defined, that is, independent of the choice of $Y$ and $z$. Also this is a conformal-invariant property of surfaces. One may compare with [Ejiri 1988b; 1988a; Kokubu 2002; Ma 2005].

We can derive that, for an $m$-isotropic surface $y$ with $m \geq 2$,

$$
\left\langle\left(D_{z}\right)^{i} \kappa,\left(D_{z}\right)^{j} \kappa\right\rangle=0 \quad \text { for } i+j \leq 2 m-3 .
$$

If $y: M \rightarrow Q_{1}^{n}$ is not contained in any $2 m$-dimensional spacelike Riemannian sphere $S^{2 m}$ of $Q_{1}^{n}$, we say that $y$ is full in $Q_{1}^{n}$.

If $y$ is $m$-isotropic and not $(m+1)$-isotropic, we say $y$ is strictly m-isotropic.

Similarly to [Ejiri 1988b; Ma 2005; Ma and Wang 2008], we can define holomorphic forms for S-Willmore spheres:

Lemma 3.2. Let $y: S^{2} \rightarrow Q_{1}^{n}$ be a strictly m-isotropic $S$-Willmore sphere. On the subset $M_{0} \subset S^{2}$ where $y$ has no umbilic points, let $Y$ be the canonical lift of $y$, and let $\hat{Y}$ be a local lift of its dual Willmore surface satisfying $\langle Y, \hat{Y}\rangle=-1$. Write $\hat{\kappa}=\hat{Y}_{z z}^{\perp}$, that is, $\hat{\kappa} \perp\left\{\hat{Y}, \hat{Y}_{z}, \hat{Y}_{\bar{z}}, \hat{Y}_{z \bar{z}}\right\}$. Then the differential form

$$
\begin{aligned}
\Omega_{m} d z^{4 m+4} & :=\left\langle Y_{z}^{(m+1)}, Y_{z}^{(m+1)}\right\rangle\left\langle\hat{Y}_{z}^{(m+1)}, \hat{Y}_{z}^{(m+1)}\right\rangle d z^{4 m+4} \\
& =\left\langle D_{z}^{(m-1)} \kappa, D_{z}^{(m-1)} \kappa\right\rangle\left\langle\hat{D}_{z}^{(m-1)} \hat{\kappa}, \hat{D}_{z}^{(m-1)} \hat{\kappa}\right\rangle d z^{4 m+4}
\end{aligned}
$$

is a globally defined holomorphic $(4 m+4)$-form on $S^{2}$, and hence vanishes. Here $D_{z}$ and $\hat{D}_{z}$ denote the normal connections of $Y$ and $\hat{Y}$.

Proof. On $M_{0}$, the formula for $\Omega_{m} d z^{4 m+4}$ is meaningful. We note that the normal connection $\hat{D}_{z}$ is equal to $D_{z}$. With respect to another complex coordinate $w$, a canonical lift is $Y_{1}=Y \cdot|d w / d z|$. So $\hat{Y}_{1}=\hat{Y} \cdot|d z / d w|$ is the lift of dual surface and $\hat{\kappa}_{1}=\hat{Y}_{1 z z}^{\perp}=\hat{\kappa} \cdot|d z / d w|^{-1} \cdot(d z / d w)^{2}$. Together with (4) and (12), $\Omega_{m} d z^{4 m+4}$ is invariant when $(Y, z)$ changes.

By using (9) and the fact that the normal connection $\hat{D}_{z}$ of $\hat{Y}$ is equal to $D_{z}$, we derive

$$
\begin{aligned}
\hat{\kappa} & =\rho \kappa, \\
D_{z} \hat{\kappa} & =\rho D_{z} \kappa+(\cdots) \kappa, \\
D_{z}^{(i)} \hat{\kappa} & =\rho D_{z}^{(i)} \kappa+(\cdots) D_{z}^{(i-1)} \kappa+\cdots+(\cdots) \kappa .
\end{aligned}
$$


By (11), we see that $\Omega_{m} d z^{4 m+4}=\rho^{2}\left\langle D_{z}^{(m-1)} \kappa, D_{z}^{(m-1)} \kappa\right\rangle^{2} d z^{4 m+4}$. To show $\Omega_{m}$ is holomorphic, with the Ricci equation (3), (11) and the S-Willmore condition, we have

$$
\begin{aligned}
\left\langle D_{z}^{(m-1)} \kappa\right. & \left., D_{z}^{(m-1)} \kappa\right\rangle_{\bar{z}}=2\left\langle D_{\bar{z}} D_{z}^{(m-1)} \kappa, D_{z}^{(m-1)} \kappa\right\rangle \\
& =2\left\langle D_{z} D_{\bar{z}}\left(D_{z}^{(m-2)} \kappa\right)-(\cdots) \kappa, D_{z}^{(m-1)} \kappa\right\rangle=\cdots \\
& =2\left\langle D_{z}^{(m-1)} D_{\bar{z}}(\kappa)+(\cdots) D_{z}^{(m-2)} \kappa+\cdots+(\cdots) \kappa, D_{z}^{(m-1)} \kappa\right\rangle \\
& =-\bar{\mu}\left\langle D_{z}^{(m-1)} \kappa, D_{z}^{(m-1)} \kappa\right\rangle .
\end{aligned}
$$

Together with (10), we see that $\Omega_{m \bar{z}} \equiv 0$.

To show $\Omega_{m}(\mathrm{~d} z)^{4 m+4}$ extends to $S^{2}$ as a holomorphic form, note that by (3) and (6),

$$
D_{\bar{z}} D_{z}^{(j)} \kappa=\cdots=D_{z}^{(j)} D_{\bar{z}} \kappa+\cdots+(\cdots) \kappa \in \operatorname{span}\left\{\kappa, \ldots, D_{z}^{(m-1)} \kappa\right\} .
$$

By Chern's lemma on pseudoholomorphic functions [1970], the zero points of $D_{z}^{(j)} \kappa$ for $1 \leq j \leq m-1$ are isolated. So $\left\{\kappa, \ldots, D_{z}^{(m-1)} \kappa\right\}$ forms a holomorphic vector bundle $F^{m}$ on $M_{0}^{\prime}=M_{0} \backslash$ isolated zero points of $D_{z}^{(j)} \kappa$ for $\left.1 \leq j \leq m-1\right\}$.

Let $\left\{\xi, \ldots, \xi^{m-1}\right\}$ be a local frame consisting of $F^{m}$ around an umbilical point $p \in S^{2} \backslash M_{0}$ such that

$$
\operatorname{span}\left\{\kappa, \ldots, D_{z}^{(i)} \kappa\right\}=\operatorname{span}\left\{\xi, \ldots, \xi^{i}\right\} \quad \text { for } i=1, \ldots, m-1 .
$$

Set $g=\left\langle\xi^{m-1}, \xi^{m-1}\right\rangle$ and $D_{z}^{(m-1)} \kappa=f \xi_{i}^{m-1} \bmod \left\{\xi, \ldots, \xi^{m-2}\right\}$. Then comparing with (13), we have $-\bar{\mu}=2 f_{\bar{z}} / f$. Together with (9) we have

$$
\rho\left\langle D_{z}^{(m-1)} \kappa, D_{z}^{(m-1)} \kappa\right\rangle=-2\langle\kappa, \bar{\kappa}\rangle f^{2} g+\bar{\mu}_{z} f^{2} g,
$$

and

$$
\bar{\mu}_{z} f^{2} g=\left(\bar{\mu} f^{2} g\right)_{z}-\bar{\mu}\left(2 f f_{z} g+f g_{z}\right)=\left(-2 f_{\bar{z}} f g\right)_{z}+2 f_{\bar{z}}\left(2 f_{z} g+f g_{z}\right),
$$

showing that $\Omega_{m}$ is a smooth function (depending on $z$ ). Then $\Omega_{m}(\mathrm{~d} z)^{4 m+4}$ extends smoothly to $S^{2} \backslash M_{0}$ as desired. It is holomorphic both on $M_{0}^{\prime}$ and in the interior of $S^{2} \backslash M_{0}$ (it vanishes in the latter case). For the same reason $\Omega_{m}(\mathrm{~d} z)^{4 m+4}$ extends smoothly to $M_{0}^{\prime} \backslash M_{0}$. So it is holomorphic on all of $S^{2}$.

The method used here is suggested by Ma and it is indeed an improvement of [Ma 2005] an [Ma and Wang 2008].

From the proof of Lemma 3.2, we know that either $y$ satisfies $\rho \equiv 0$, forcing the dual surface of $y$ to reduce to a point, or that $y$ is a totally isotropic surface in $Q_{1}^{n}$. In the case that $y$ is totally isotropic, if $y$ is in some Riemannian sphere $S^{2 m} \subset Q_{1}^{n}$, we have this: 
Proposition 3.3 [Ejiri 1988b]. If y is totally isotropic and contained in some Riemannian sphere $S^{2 m} \subset Q_{1}^{n}$, it must be a super Willmore surface in $S^{2 m}$.

If $y$ is not contained in any Riemannian sphere, it must be full in some $Q_{1}^{2 m}$. For this case, Proposition 4.3 gives the description of such surface; see Section 4 for details. Summing up, we finish the proof of Theorem C.

\section{Polar surfaces of $m$-isotropic S-Willmore surfaces in $Q_{1}^{2 m+2}$}

Next we deal with $m$-isotropic S-Willmore surfaces that are full in $Q_{1}^{2 m+2}$. Let $y: M \rightarrow Q_{1}^{2 m+2}$ be such a surface. Then by (11), we can suppose that

$$
\begin{aligned}
\kappa & =E_{1}, \\
D_{z} E_{1} & =E_{2}+\alpha_{1} E_{1} \quad \text { with } E_{2} \perp\left(E_{1}, \bar{E}_{1}\right),
\end{aligned}
$$

$$
\begin{aligned}
D_{z} E_{m-1}= & E_{m}+\alpha_{m-1} E_{m-1}+\cdots+(\cdots) E_{1}, \\
& \quad \text { with } E_{m} \perp\left(E_{1}, \ldots, E_{m-1}, \bar{E}_{1}, \ldots, \bar{E}_{m-1}\right) .
\end{aligned}
$$

So $\left\langle E_{j}, E_{j}\right\rangle=0$ for $1 \leq j \leq m-1$. We note that on the points that $E_{i}=0$, by a similar treatment as before, $E_{i}$ can be extended to the whole surface since it is defined on a open dense subset.

To give the definition of polar surfaces, we still need more preparation. By the S-Willlmore condition,

$$
D_{\bar{z}} E_{1}=-\frac{1}{2} \mu E_{1} \text {. }
$$

Together with the Ricci equation, we have

$$
\begin{aligned}
D_{\bar{z}} E_{2} & =D_{\bar{z}} D_{z} E_{1}-D_{\bar{z}}\left((\cdots) E_{1}\right)=D_{z} D_{\bar{z}} E_{1} \bmod \left(E_{1}\right) \\
& =-\frac{1}{2} \bar{\mu} E_{2} \bmod \left(E_{1}\right) .
\end{aligned}
$$

Using Ricci equations repeatedly, we get

$$
D_{\bar{z}} E_{i}=-\frac{1}{2} \bar{\mu} E_{i} \quad \bmod \left(E_{1}, \ldots, E_{i-1}\right) \quad \text { for } 1 \leq i \leq m .
$$

Then we have that, for all $1 \leq j \leq m-1$,

$$
\left\langle E_{j}, \bar{E}_{j}\right\rangle_{z}=\left\langle D_{z} E_{j}, \bar{E}_{j}\right\rangle+\left\langle E_{j}, D_{z} \bar{E}_{j}\right\rangle=\left(\alpha_{j}-\frac{1}{2}(\mu)\right)\left\langle E_{j}, \bar{E}_{j}\right\rangle,
$$

Applying Chern's lemma about almost holomorphic functions [1970], we see that either $\left\langle E_{i}, \bar{E}_{i}\right\rangle \equiv 0$ on a open dense subset $M_{0}$ of $M$, or $\left\langle E_{i}, \bar{E}_{i}\right\rangle$ has only isolated zero points. We will show that if $\left\langle E_{j}, \bar{E}_{j}\right\rangle \equiv 0$ on $M_{0}$, then $y$ must be contained in some $Q_{1}^{2 j+2}$.

To see this, we need two lemmas. Let $\mathbb{C}_{1}^{n}$ be the complexification of $R_{1}^{n}$ with its inner product. The first one is about isotropic vector in $\mathbb{C}_{1}^{n}$. 
Lemma 4.1. Let $E$ be a nonzero vector in $\mathbb{C}_{1}^{n}$ with $\langle E, E\rangle=0$, E must either equal to $\lambda\left(e_{1}+i e_{2}\right)$ with $e_{1}, e_{2} \in R_{1}^{n},\left\langle e_{1}, e_{2}\right\rangle=0,\left\langle e_{1}, e_{1}\right\rangle=\left\langle e_{2}, e_{2}\right\rangle=1$, and $\langle E, \bar{E}\rangle \neq 0$; or equal to $\lambda\left(e_{1}-e_{2}\right)$ with $e_{1}, e_{2} \in R_{1}^{n}$ and $\left\langle e_{1}, e_{2}\right\rangle=0,\left\langle e_{1}, e_{1}\right\rangle=-\left\langle e_{2}, e_{2}\right\rangle=1$, and $\langle E, \bar{E}\rangle=0$.

The other concerns complex functions:

Lemma 4.2. Let $\gamma$ be a complex function such that $\gamma_{\bar{z}}=\bar{\gamma}_{z}$. There must be a real function $f=f(z, \bar{z})$ satisfying $\gamma=f_{z}$.

Proof. Set $\gamma=\gamma_{1}+i \gamma_{2}$ and $z=u+i v$, where $\gamma_{1}, \gamma_{2}, u, v$ are real. Putting these in the formula $\gamma_{\bar{z}}=\bar{\gamma}_{z}$ and expanding it shows $\partial \gamma_{1} / \partial v=-\partial \gamma_{2} / \partial u$, which is just the integrability condition of the PDE

$$
\frac{\partial f}{\partial u}=\gamma_{1} \quad \text { and } \quad \frac{\partial f}{\partial v}=-\gamma_{2} .
$$

Proposition 4.3. Let $y: M \rightarrow Q_{1}^{2 m+2}$ be an $(m+1)$-isotropic $S$-Willmore surface that is full in $Q_{1}^{2 m+2}$. Then y induces two $m$-isotropic $S$-Willmore surfaces $[\hat{L}]$ and $[\hat{R}]$ in $Q_{1}^{2 m+2}$, called polar surfaces of $y$. One of these polar surfaces reduces to a point and the other is conformal to an $m$-isotropic complete spacelike stationary $(H=0)$ surface in $R_{1}^{2 m+2}$. Both $y$ and its dual surface are polar surfaces of the nondegenerate polar surface.

Proof. Since $y$ is $(m+1)$-isotropic and full in $Q_{1}^{2 m+2}$, it is totally isotropic and its derivatives $Y_{z}^{(k)}$ give $(m+1)$ independent isotropic vectors in $\mathbb{C}_{2}^{m+2}$. Using Lemma 4.1, we can suppose that $\left\langle E_{j}, \bar{E}_{j}\right\rangle \equiv 0$ for some $j$. Then $E_{j}=\lambda_{j}\left(e_{j 1}-e_{j 2}\right)$ for two orthogonal vector fields $e_{j 1}, e_{j 2} \in V$ such that $\left\langle e_{j 1}, e_{j 2}\right\rangle=0$ and $\left\langle e_{j 1}, e_{j 1}\right\rangle=$ $-\left\langle e_{j 2}, e_{j 2}\right\rangle=1$. Write

$$
L_{j}=\frac{1}{\sqrt{2}}\left(e_{j 1}-e_{j 2}\right), \quad R_{j}=\frac{1}{\sqrt{2}}\left(e_{j 1}+e_{j 2}\right) \quad\left(\text { which implies } E_{j}=\sqrt{2} \lambda_{j} L_{j}\right) .
$$

If $j=1$, we have

$$
\left\{\begin{array}{l}
\left(L_{1}\right)_{z}=\alpha_{11} L_{1}+\psi_{11}, \\
\left(R_{1}\right)_{z}=-\alpha_{11} R_{1}+\psi_{12}-\bar{\mu}\left\langle R_{1}, E_{1}\right\rangle Y-2\left\langle R_{1}, E_{1}\right\rangle Y_{\bar{z}}, \\
D_{\bar{z}} D_{z} \psi-D_{z} D_{\bar{z}} \psi=0 \quad \text { for all } \psi \in V^{\perp} \text { and } \psi \perp\left\{L_{1}, R_{1}\right\} .
\end{array}\right.
$$

Here $\psi_{11}, \psi_{12} \in V_{\mathbb{C}}^{\perp}$ and $\psi_{11}, \psi_{12} \perp\left\{L_{1}, R_{1}\right\}$. Since $D_{\bar{z}} \kappa \| \kappa$, we have $\psi_{11}=0$ and $\left(L_{1}\right)_{z}=\alpha_{11} L_{1}$. So $\left[L_{1}\right]$ is a point in $Q_{1}^{2 m+2}$. Together with the third formula in (16), we can choose new $L_{1}$ and $e_{13}, \ldots, e_{1,2 m}$ so that $\alpha_{11}=0$ and $e_{13}, \ldots, e_{1,2 m}$ is a parallel orthonormal frame of $V^{\perp} \bmod L_{1}, R_{1}$. So

$$
\begin{aligned}
& \left(R_{1}\right)_{z}=\sum_{k} \lambda_{1 k} e_{1 k}-\bar{\mu}\left\langle R_{1}, E_{1}\right\rangle Y-2\left\langle R_{1}, E_{1}\right\rangle Y_{\bar{z}} . \\
& \left(e_{1 k}\right)_{z}=-\frac{1}{2} \lambda_{1 k} L_{1} \quad \text { for } k=3, \ldots, 2 m, \text { with } \lambda_{1 k} \text { functions. }
\end{aligned}
$$


The Ricci equations lead to $\lambda_{1 k \bar{z}}=\bar{\lambda}_{1 k z}$ for $k=3, \ldots, 2 m$. Using Lemma 4.2, we see that there exist real functions $f_{1 k}$ such that $\lambda_{1 k}=f_{1 k z}$. Set

$$
\hat{R}_{1}=R_{1}-\sum_{k} f_{1 k} e_{1 k}-\frac{1}{2}\left(\sum_{k} f_{1 k}\right) L_{1} .
$$

Differentiating (17), we obtain

$$
\left(\hat{R}_{1}\right)_{z}=-\bar{\mu}\left\langle R_{1}, E_{1}\right\rangle Y-2\left\langle R_{1}, E_{1}\right\rangle Y_{\bar{z}} .
$$

Thus $Y$ is located in a 6-dimensional subspace $\mathbb{R}_{2}^{6} \subset R_{2}^{2 m+4}$. By the condition that $y$ is full, we have $j=m=1$.

From [Ma and Wang 2008], we see that $\hat{R}_{1}$ is conformal to a stationary surface in $\mathbb{R}_{1}^{4}$, and that $[Y]$ and its dual surface $[\hat{Y}]$ are just polar transforms of $\hat{R}_{1}$. In fact, we see directly that $L_{1}$ is in the central sphere of $\hat{R}_{1}$ and dual surface of $\hat{R}_{1}$. So $\hat{R}_{1}$ is a Willmore surface with dual surface reducing to a point and thus is S-Willmore.

If $j>1$, by (15) and (14), we have $\left(L_{j}\right)_{z}=\alpha_{j 1} L_{j}$ for some function $\alpha_{j 1}$. This means that $\left[L_{j}\right]$ is a point in $Q_{1}^{2 m+2}$ and we can choose new $L_{j}$ and $R_{j}$ such that $\left(L_{j}\right)_{z}=0$. Differentiating $\left\langle R_{j}, E_{i}\right\rangle=0$, we have

$$
0=\left\langle\left(R_{j}\right)_{z}, E_{i}\right\rangle+\left\langle R_{j},\left(E_{i}\right)_{z}\right\rangle=\left\langle\left(R_{j}\right)_{z}, E_{i}\right\rangle-\sqrt{2} \lambda_{j, j-1} \delta_{i, j-1},
$$

where $\lambda_{j, j-1}$ is some function. So we can assume that

$$
\left(R_{j}\right)_{z}=\lambda_{j, j-1} E_{j-1}+\psi_{j+1,2},
$$

where $\psi_{j+1,2} \in V_{\mathbb{C}}^{\perp}$ with $\psi_{j+1,2} \perp\left\{E_{i}, \bar{E}_{i}, L_{j}, R_{j}, 1 \leq i<j\right\}$. In summary, we have

$$
\begin{aligned}
\left(E_{i}\right)_{z} & =\alpha_{i} E_{i}+E_{i+1} \quad \text { for } 1 \leq i<j, \\
\left(\bar{E}_{1}\right)_{z} & =-\frac{1}{2} \mu \bar{E}_{1}-2 \bar{\mu}\left\langle E_{1}, \bar{E}_{1}\right\rangle Y-2\left\langle E_{1}, \bar{E}_{1}\right\rangle Y_{\bar{z}}, \\
\left(\bar{E}_{i}\right)_{z} & =-\frac{1}{2} \mu \bar{E}_{i}-\left\langle E_{i}, \bar{E}_{i}\right\rangle /\left\langle E_{i-1}, \bar{E}_{i-1}\right\rangle \bar{E}_{i-1} \quad \text { for } 1<i<j, \\
\left(L_{j}\right)_{z} & =0 \\
\left(R_{j}\right)_{z} & =\lambda_{j, j-1} E_{j-1}+\psi_{j+1,2}, \\
\psi_{z} & =D_{z} \psi \quad \text { for all } \psi \in V^{\perp} \text { with } \psi \perp\left\{\operatorname{Re}\left(E_{i}\right), \operatorname{Im}\left(E_{i}\right), L_{j}, R_{j}, 1 \leq i<j\right\} .
\end{aligned}
$$

That $D_{\bar{z}} D_{z} \psi=D_{z} D_{\bar{z}} \psi$ shows that we can choose $\left\{e_{j, 2 j+1}, \ldots, e_{j, 2 m}\right\}$ as a parallel orthonormal frame of $V^{\perp} \bmod \left\{\operatorname{Re}\left(E_{i}\right), \operatorname{Im}\left(E_{i}\right), L_{j}, R_{j}, 1 \leq i<j\right\}$. So

$$
\left(R_{j}\right)_{z}=\lambda_{j, j-1} \bar{E}_{j-1}+\Sigma_{k} \lambda_{j k} e_{j, 2 j+k},
$$

where $\lambda_{j k \bar{z}}=\bar{\lambda}_{j k z}$. Using Lemma 4.2, we see that there exist real functions $f_{j k}$ such that $f_{j k z}=\lambda_{j k}$. Setting

$$
\hat{R}_{j}=R_{j}-\sum_{k} f_{j k} e_{j, 2 j+k}-\left(\sum_{k} f_{j k}\right) L_{j} .
$$


shows that $\left(\hat{R}_{j}\right)_{z}=\lambda_{j, j-1} \bar{E}_{j-1}$.

So $Y$ is located in a $(2 j+4)$-dimensional subspace $\mathbb{R}_{2}^{2 j+4} \subset \mathbb{R}_{2}^{2 m+4}$. By the condition that $y$ is full, $j=m$.

From $\left(\hat{R}_{m}\right)_{z \bar{z}}=(\cdots) L_{m} \bmod \left\{\hat{R}, \hat{R}_{z}, \hat{R}_{\bar{z}}\right\}$, we see that $L_{m}$ is in the central sphere of $\hat{R}_{m}$ and dual surface of $\hat{R}_{1}$. The methods of [Ma and Wang 2008] show that $\hat{R}_{m}$ is conformal to a stationary surface in $\mathbb{R}_{1}^{2 m+2}$. So $\hat{R}_{m}$ is a S-Willmore surface with dual surface reducing to a point.

Following [Ma and Wang 2008], we call $\left[\hat{R}_{m}\right],\left[L_{m}\right]: S^{2} \rightarrow Q_{1}^{2 m+2}$ polar surfaces of $y=[Y]: S^{2} \rightarrow Q_{1}^{2 m+2}$ for all $m \geq 1$.

To see that $\hat{R}_{m}$ is $m$-isotropic and $y$ together with its dual surface $[\hat{Y}]$ are just its polar surfaces, let us calculate $\hat{R}_{z z}, \ldots, \hat{R}_{m z}^{(m+2)}$ :

$$
\begin{aligned}
\left(\hat{R}_{m}\right)_{z z} & =(\cdots) \bar{E}_{m-2} \bmod \left\{\hat{R}, \hat{R}_{z}\right\}, \\
& \vdots \\
\left(\hat{R}_{m}^{(m-1)}\right)_{z} & =(\cdots) \bar{E}_{1} \bmod \left\{\hat{R}, \hat{R}_{z}, \bar{E}_{m-1}, \ldots, \bar{E}_{2}\right\}, \\
\left(\hat{R}_{m}^{(m)}\right)_{z} & =(\cdots)\left(Y_{\bar{z}}+\frac{1}{2} \bar{\mu} Y\right) \bmod \left\{\hat{R}, \hat{R}_{z}, \bar{E}_{m-1}, \ldots, \bar{E}_{1}\right\}, \\
\left(\hat{R}_{m}^{(m+1)}\right)_{z} & =(\cdots)(\rho Y+\hat{Y}) \bmod \left\{\hat{R}, \hat{R}_{z}, \bar{E}_{m-1}, \ldots, \bar{E}_{1}, Y_{\bar{z}}+\frac{1}{2} \bar{\mu} Y\right\} .
\end{aligned}
$$

So $\hat{R}_{m}$ is $m$-isotropic. [Y] and $[\hat{Y}]=\left[\hat{Y}=N+\bar{\mu} Y_{z}+\mu Y_{\bar{z}}+\frac{1}{2}|\mu|^{2} Y\right]$ are polar surfaces of $\hat{R}_{m}$.

From the proof of Proposition 4.3, we see that to define the polar surfaces we just need $y$ to be $m$-isotropic and full in $Q_{1}^{2 m+2}$. Let $y: M \rightarrow Q_{1}^{2 m+2}$ be such an SWillmore surface. On an open dense subset we have $\left\langle E_{j}, \bar{E}_{j}\right\rangle \neq 0$ for $1 \leq j \leq m-1$. So the complement of $\operatorname{span}\left\{\operatorname{Re} E_{j}, \operatorname{Im} E_{j}\right\}$ in $V^{\perp}$ is a Lorentzian 2-bundle. Let $\{L, R\}$ be a null basis of it, that is, $\langle L, L\rangle=\langle R, R\rangle=0$ and $\langle L, R\rangle=-1$, with orientation restrictions $[L],[R]: \tilde{M} \rightarrow Q_{1}^{2 m+2}$ well defined.

Proposition 4.4. Let $y: M \rightarrow Q_{1}^{2 m+2}$ be an $m$-isotropic $S$-Willmore surface that is full in $Q_{1}^{2 m+2}$. Then y induces two $m$-isotropic $S$-Willmore surfaces $[L]$ and $[R]$ in $Q_{1}^{2 m+2}$, called the polar surfaces of $y$ (they may degenerate). Also y and its dual surface are polar surfaces of the nondegenerate polar surface.

Remark 4.5. In the case $m=1$, the normal bundle $V^{\perp}$ is just a Lorentzian 2bundle with two canonical lightlike directions, which enables us to define polar surfaces naturally. See [Ma and Wang 2008] for details.

Corollary 4.6. Let y be a surface belonging to both case (i) and case (iii) in Theorem $C$. Then $y$ is conformal to a totally isotropic complete spacelike stationary surface in $R_{1}^{2 m}$. 
Remark 4.7. It is natural to ask that whether there are nontrivial S-Willmore spheres belonging to case (iii). For the case $m=1$, the answer is negative; see the next section. For the case in $R_{1}^{6}$ we can give examples of spheres both of case (i) and case (iii). See the example below.

Example 4.8. Define

$$
f: M=\mathbb{C} \backslash\{0,1\} \rightarrow \mathbb{C}_{1}^{6}, \quad z \mapsto z v_{0}+\frac{v_{1}}{z}+\frac{v_{2}}{z-1}
$$

with $v_{1}=(1, i, 0,0,0,0), v_{2}=(0,0,1, i, 0,0)$ and $v_{0}=v_{1}+v_{2}+(0,0,0,0,1,1)$. Then $y=2 \operatorname{Re} f$ is just a totally isotropic complete spacelike stationary surface, and its compactification is a Willmore sphere with $W(y)=8 \pi$. Its polar surface $l$ corresponds to a Willmore sphere with $W(y)=8 \pi$ and can be written as

$$
\begin{aligned}
l=2 \operatorname{Re}(\tilde{l}), \quad \tilde{l} & =\frac{1}{3}\left(\left(\frac{1}{z}+\frac{1}{z-1}\right) \tilde{v}_{0}+\left(z+1+\frac{1}{z-1}\right) \tilde{v}_{1}-\left(z-2+\frac{1}{z}\right) \tilde{v}_{2}\right) \\
& =\frac{1}{3}\left(z\left(\tilde{v}_{1}-\tilde{v}_{2}\right)+\frac{1}{z}\left(\tilde{v}_{0}-\tilde{v}_{2}\right)+\frac{1}{z-1}\left(\tilde{v}_{0}+\tilde{v}_{1}\right)+\left(\tilde{v}_{1}+2 \tilde{v}_{2}\right)\right),
\end{aligned}
$$

with $\tilde{v}_{0}=(-1,0,0,0,0,1), \quad \tilde{v}_{1}=(0,1,-i, 0,0,0)$ and $\tilde{v}_{2}=(0,0,0,1,-i, 0)$. So they are both spacelike stationary surfaces of the type

$$
x=\operatorname{Re}\left(\frac{v_{1}}{z-\lambda_{1}}+\frac{v_{1}}{z-\lambda_{2}}+\frac{v_{1}}{z-\lambda_{3}}\right),
$$

where the $v_{j}$ are orthogonal isotropic vectors and the $\lambda_{j}$ are distinct complex numbers such that the surfaces are complete.

Remark 4.9. It is desirable to have examples that fall under case (iii) and but not case (i). One way to find these follows Bryant's methods [1984] and is used in Example 4.8. Alternatively, see [Ejiri 1993] and [Kokubu 2002] for two iterative constructions of $m$-isotropic minimal surfaces. Both methods require detailed technical computations, and so far we have not succeeded in using them.

\section{Null-umbilic S-Willmore spheres in $Q_{1}^{4}$}

Let $y: S^{2} \rightarrow Q_{1}^{4}$ be a null-umbilic S-Willmore spheres in $Q_{1}^{4}$. Recall that $y$ is called null-umbilic if $\kappa$ is a complexified lightlike section, that is, $\langle\kappa, \kappa\rangle=0$. Then the structure equations simplify to

$$
\begin{array}{lll}
Y_{z z}=-\frac{1}{2} s Y+\lambda_{1} L, & N_{z}=-s Y_{\bar{z}}+2 \lambda_{1 \bar{z}} L, \quad L_{z}=0, \\
Y_{z \bar{z}}=\frac{1}{2} N, & R_{z}=\lambda_{1 \bar{z}} Y+2 \lambda_{1} Y_{\bar{z}}
\end{array}
$$

for some local function $\lambda_{1}$ and normal frame $L, R$. The Gauss equation shows that $s_{\bar{z}}=0$. Let the complex coordinate $z$ transform projectively. Then $s(d z)^{2}$ is a globally defined holomorphic 2-form on $S^{2}$, and $s \equiv 0$. The only nontrivial invariant left is $\lambda_{1}$, and the only remaining integrability condition to be satisfied is 
$\left(\lambda_{1}\right)_{\bar{z} \bar{z}} \equiv 0$ on the whole $S^{2}=\mathbb{C P}^{1}$. It is easy to see that on $\mathbb{C}$ the general solution to this equation takes the form $\lambda_{1}(z, \bar{z})=A(z) \cdot \bar{z}+B(z)$, where $A(z)$ and $B(z)$ are holomorphic functions. With respect to the new coordinate $w=1 / z$, we find that

$$
\lambda_{1}=\left(A\left(\frac{1}{w}\right) \frac{1}{\bar{w}}+B\left(\frac{1}{w}\right)\right) \cdot \frac{\bar{w}}{w^{3}} .
$$

The poles will show up unless $A(z)=B(z) \equiv 0$ and $\lambda_{1} \equiv 0$ identically. Hence we have proved this:

Proposition 5.1. Let $y: M \rightarrow Q_{1}^{4}$ be an immersed spacelike Willmore 2-sphere that is also null-umbilic. Then $y(M)$ is congruent to a round 2-sphere.

Remark 5.2. Leitner [2000] showed that compact null-umbilic surfaces in $R_{1}^{4}$, $S_{1}^{4}$ or $H_{1}^{4}$ must be topological 2-spheres and be certain deformations of round 2-spheres.

Our conclusion does not mean that there are no nontrivial compact twistorial surfaces in $Q_{1}^{4}$. It should be understood that there exist many them, but all have branch points. Note that there are many stationary surfaces in $R_{1}^{4}$ with planar ends, and polar transforms of surfaces provide null-umbilic Willmore surfaces (which may have branch points).

\section{Acknowledgments}

The author is thankful to Professor Xiang Ma for valuable discussions and suggestions, and also thanks the referees for valuable suggestions.

\section{References}

[Alías and Palmer 1996] L. J. Alías and B. Palmer, "Conformal geometry of surfaces in Lorentzian space forms", Geom. Dedicata 60:3 (1996), 301-315. MR 97f:53099 Zbl 0863.53037

[Blaschke 1929] W. Blaschke, Vorlesungen über Differentialgeometrie und geometrische Grundlagen von Einsteins Relativitätstheorie, III: Differentialgeometrie der Kreise und Kugeln, Springer, Berlin, 1929. JFM 55.0422.01

[Bryant 1984] R. L. Bryant, "A duality theorem for Willmore surfaces", J. Differential Geom. 20:1 (1984), 23-53. MR 86j:58029 Zbl 0555.53002

[Burstall et al. 2002] F. Burstall, F. Pedit, and U. Pinkall, "Schwarzian derivatives and flows of surfaces", pp. 39-61 in Differential geometry and integrable systems (Tokyo, 2000), edited by M. Guest et al., Contemp. Math. 308, Amer. Math. Soc., Providence, RI, 2002. MR 2004f:53010 Zbl 1031.53026

[Chern 1970] S. S. Chern, "On the minimal immersions of the two-sphere in a space of constant curvature”, pp. 27-40 in Problems in analysis (Princeton, 1969), edited by R. C. Gunning, Princeton Univ. Press, 1970. MR 50 \#14593 Zbl 0217.47601

[Ejiri 1988a] N. Ejiri, "Isotropic harmonic maps of Riemann surfaces into the de Sitter space time", Quart. J. Math. Oxford Ser. (2) 39:155 (1988), 291-306. MR 89m:58047 Zbl 0663.58007

[Ejiri 1988b] N. Ejiri, "Willmore surfaces with a duality in $S^{N}(1)$ ", Proc. London Math. Soc. (3) 57:2 (1988), 383-416. MR 89h:53117 Zbl 0671.53043 
[Ejiri 1993] N. Ejiri, "A Darboux theorem for null curves in $\mathbb{C}^{2 m+1 ", ~ p p . ~ 209-210 ~ i n ~ G e o m e-~}$ try and global analysis (Sendai, 1993), edited by T. Kotake et al., Tohoku Univ., Sendai, 1993. MR 1361183 Zbl 0954.53005

[Kokubu 2002] M. Kokubu, "On isotropic minimal surfaces in Euclidean space”, pp. 155-171 in Minimal surfaces, geometric analysis and symplectic geometry (Baltimore, 1999), edited by K. Fukaya et al., Adv. Stud. Pure Math. 34, Math. Soc. Japan, Tokyo, 2002. MR 2003i:53010 Zbl 1035.53017

[Leitner 2000] F. Leitner, "Twistorial construction of spacelike surfaces in Lorentzian 4-manifolds", pp. 113-135 in Geometry and topology of submanifolds X (Beijing/Berlin, 1999), edited by W. H. Chen et al., World Sci. Publ., River Edge, NJ, 2000. MR 2001k:53091 Zbl 0984.53022

[Ma 2005] X. Ma, Willmore surfaces in $S^{n}$ : transforms and vanishing theorems, thesis, Technischen Universität Berlin, 2005, Available at http://edocs.tu-berlin.de/diss/2005/ma_xiang.pdf.

[Ma 2006] X. Ma, "Adjoint transform of Willmore surfaces in $\mathbb{S}^{n} "$, Manuscripta Math. 120:2 (2006), 163-179. MR 2007k:53083 Zbl 1116.53013

[Ma and Wang 2008] X. Ma and P. Wang, "Spacelike Willmore surfaces in 4-dimensional Lorentzian space forms”, Sci. China Ser. A 51:9 (2008), 1561-1576. MR 2009h:53122 Zbl 1163.53005

[Montiel 2000] S. Montiel, "Willmore two-spheres in the four-sphere", Trans. Amer. Math. Soc. 352:10 (2000), 4469-4486. MR 2001b:53080 Zbl 0961.53035

[Musso 1990] E. Musso, "Willmore surfaces in the four-sphere", Ann. Global Anal. Geom. 8:1 (1990), 21-41. MR 92g:53059 Zbl 0705.53028

[O’Neill 1983] B. O’Neill, Semi-Riemannian geometry, with applications to relativity, Pure and Applied Mathematics 103, Academic, New York, 1983. MR 85f:53002 Zbl 0531.53051

[Thomsen 1923] G. Thomsen, "Über konforme Geometrie I: Grundlagen der konformen Flächentheorie”, Abh. Math. Sem. Univ. Hamburg (1923), 31-56. JFM 49.0530 .02

[Wang 1998] C. Wang, "Moebius geometry of submanifolds in $S^{n}$ ", Manuscripta Math. 96:4 (1998), 517-534. MR 2000a:53019 Zbl 0912.53012

Received May 15, 2009. Revised September 5, 2009.

PENG WANG

DEPARTMENT OF MATHEMATICS

TONGJI UNIVERSITY

SHANGHAI 200092

CHINA

netwangpeng@tongji.edu.cn 


\title{
PACIFIC JOURNAL OF MATHEMATICS
}

\author{
http://www.pjmath.org \\ Founded in 1951 by \\ E. F. Beckenbach (1906-1982) and F. Wolf (1904-1989)
}

\section{EDITORS}

V. S. Varadarajan (Managing Editor)

Department of Mathematics

University of California

Los Angeles, CA 90095-1555

pacific@math.ucla.edu

Vyjayanthi Chari

Department of Mathematics

University of California

Riverside, CA 92521-0135

chari@math.ucr.edu

Robert Finn

Department of Mathematics Stanford University

Stanford, CA 94305-2125

finn@math.stanford.edu

Kefeng Liu

Department of Mathematics

University of California

Los Angeles, CA 90095-1555

liu@math.ucla.edu
Paulo Ney de Souza, Production Manager

ACADEMIA SINICA, TAIPEI

CALIFORNIA INST. OF TECHNOLOGY

INST. DE MATEMÁTICA PURA E APLICADA

KEIO UNIVERSITY

MATH. SCIENCES RESEARCH INSTITUTE

NEW MEXICO STATE UNIV.

OREGON STATE UNIV.

\section{Darren Long}

Department of Mathematics

University of California

Santa Barbara, CA 93106-3080

long@math.ucsb.edu

Jiang-Hua Lu

Department of Mathematics

The University of Hong Kong

Pokfulam Rd., Hong Kong

jhlu@maths.hku.hk

Alexander Merkurjev

Department of Mathematics

University of California

Los Angeles, CA 90095-1555

merkurev@math.ucla.edu

\section{PRODUCTION}

pacific@math.berkeley.edu

Silvio Levy, Senior Production Editor

\section{SUPPORTING INSTITUTIONS}

STANFORD UNIVERSITY
UNIV. OF BRITISH COLUMBIA
UNIV. OF CALIFORNIA, BERKELEY
UNIV. OF CALIFORNIA, DAVIS
UNIV. OF CALIFORNIA, LOS ANGELES
UNIV. OF CALIFORNIA, RIVERSIDE
UNIV. OF CALIFORNIA, SAN DIEGO
UNIV. OF CALIF., SANTA BARBARA

Sorin Popa

Department of Mathematics University of California

Los Angeles, CA 90095-1555 popa@math.ucla.edu

Jie Qing

Department of Mathematics

University of California

Santa Cruz, CA 95064

qing@cats.ucsc.edu

Jonathan Rogawski

Department of Mathematics

University of California

Los Angeles, CA 90095-1555

jonr@math.ucla.edu

Matthew Cargo, Production Editor

UNIV. OF CALIF., SANTA CRUZ

UNIV. OF MONTANA

UNIV. OF OREGON

UNIV. OF SOUTHERN CALIFORNIA

UNIV. OF UTAH

UNIV. OF WASHINGTON

WASHINGTON STATE UNIVERSITY

These supporting institutions contribute to the cost of publication of this Journal, but they are not owners or publishers and have no responsibility for its contents or policies.

See inside back cover or www.pjmath.org for submission instructions.

The subscription price for 2010 is US \$420/year for the electronic version, and \$485/year for print and electronic.

Subscriptions, requests for back issues from the last three years and changes of subscribers address should be sent to Pacific Journal of Mathematics, P.O. Box 4163, Berkeley, CA 94704-0163, U.S.A. Prior back issues are obtainable from Periodicals Service Company, 11 Main Street, Germantown, NY 12526-5635. The Pacific Journal of Mathematics is indexed by Mathematical Reviews, Zentralblatt MATH, PASCAL CNRS Index, Referativnyi Zhurnal, Current Mathematical Publications and the Science Citation Index.

The Pacific Journal of Mathematics (ISSN 0030-8730) at the University of California, c/o Department of Mathematics, 969 Evans Hall, Berkeley, CA 94720-3840, is published monthly except July and August. Periodical rate postage paid at Berkeley, CA 94704, and additional mailing offices. POSTMASTER: send address changes to Pacific Journal of Mathematics, P.O. Box 4163, Berkeley, CA 94704-0163.

PJM peer-review and production is managed by EditFLOW ${ }^{\mathrm{TM}}$ from Mathematical Sciences Publishers.

PUBLISHED BY PACIFIC JOURNAL OF MATHEMATICS

at the University of California, Berkeley $94720-3840$

A NON-PROFIT CORPORATION

Typeset in LATEX

Copyright $@ 2010$ by Pacific Journal of Mathematics 


\section{PACIFIC JOURNAL OF MATHEMATICS}

Volume $246 \quad$ No. $2 \quad$ June 2010

Geometric structures associated to a contact metric $(\kappa, \mu)$-space

Beniamino CAPPELLETTI MONTANO and LUigia Di TERLizzi

Multilinear singular operators with fractional rank

Ciprian Demeter, Malabika PramaniK and Christoph Thiele

A new proof of Reifenberg's topological disc theorem

GUANGHAO HONG and LiHE WANG

Global classical solutions to hyperbolic geometric flow on Riemann surfaces

FAGUi LiU and YUANZHANG ZHANG

An extension of the quintuple product identity and its applications

ZHI-GUO LIU

A generalization of the Pontryagin-Hill theorems to projective modules over

Prüfer domains

JORGE MACÍAS-DíAZ

Elliptic pseudodifferential equations and Sobolev spaces over $p$-adic fields

J. J. RodRíGUEZ-VEGA and W. A. ZÚÑIGA-GALINDO

Absolutely isolated singularities of holomorphic maps of $\mathbb{C}^{n}$ tangent to the identity

\section{FENG RONG}

Pullbacks of Eisenstein series from $\mathrm{GU}(3,3)$ and critical $L$-values for

$\mathrm{GSp}(4) \times \mathrm{GL}(2)$

\section{ABHISHEK SAHA}

Isomorphism invariants of restricted enveloping algebras

HAMID USEFI

Spacelike S-Willmore spheres in Lorentzian space forms

PENG WANG 